\title{
X-ray Structure of a Maquette Scaffold
}

\section{Steve S. Huang, Brian R. Gibney, Steven E. Stayrook, P. Leslie Dutton and Mitchell Lewis*}

The Johnson Research

Foundation, Department of

Biochemistry and Biophysics

School of Medicine, University

of Pennsylvania, 37th and

Hamilton Walk, Philadelphia

PA 19102-6059, USA

\begin{abstract}
Maquettes are de novo designed mimicries of nature used to test the construction and engineering criteria of oxidoreductases. One type of scaffold used in maquette construction is a four- $\alpha$-helical bundle. The sequence of the four- $\alpha$-helix bundle maquettes follows a heptad repeat pattern typical of left-handed coiled-coils. Initial designs were molten globular due partly to the minimalist approach taken by the designers. Subsequent iterative redesign generated several structured scaffolds with similar heme binding properties. Variant $\left[\mathrm{I}_{6} \mathrm{~F}_{13}\right]_{2}$, a structured scaffold, was partially resolved with NMR spectroscopy and found to have a set of mobile inter-helical packing interfaces. Here, the $\mathrm{X}$-ray structure of a similar peptide $\left(\left[\mathrm{I}_{6} \mathrm{~F}_{13} \mathrm{M}_{31}\right]_{2}\right.$ i.e. $\left\{[C G G G \text { EIWKL HEEFLKK FEELLKL HEERLKKM }]_{2}\right\}_{2}$ which we call L31M), has been solved using MAD phasing and refined to $2.8 \AA$ resolution. The structure shows that the maquette scaffold is an anti-parallel four-helix bundle with "up-up-down-down" topology. No pre-formed heme-binding pocket exists in the protein scaffold. We report unexpected inter-helical crossing angles, residue positions and translations between the helices. The crossing angles between the parallel helices are $-5^{\circ}$ rather than the expected $+20^{\circ}$ for typical left-handed coiled-coils. Deviation of the scaffold from the design is likely due to the distribution and size of hydrophobic residues. The structure of L31M points out that four identical helices may interact differently in a bundle and heptad repeats with an alternating [HPPHHPP]/[HPPHHPH] $(\mathrm{H}$ : hydrophobic, P: polar) pattern are not a sufficient design criterion to generate left-hand coiled-coils.
\end{abstract}

(C) 2003 Elsevier Science Ltd. All rights reserved

Keywords: four-helix bundle; X-ray crystallography; de novo protein design; MAD phasing

\section{Introduction}

In the last decade, de novo protein design has emerged as a constructive methodology for testing our understanding of the relationship between protein sequence, structure and function. ${ }^{1-6}$ Fourhelix-bundle proteins are ubiquitous in nature and serve as convenient scaffolds for the construction of simplified functional versions of complex natural enzymes or molecular maquettes. We have designed a series of maquettes to allow us to study how large multi-heme proteins function in

Present address: B. R. Gibney, Department of Chemistry, Columbia University, New York, NY 10027, USA.

Abbreviations used: MAD, multiple anomalous dispersion; NMR, nuclear magnetic resonance.

E-mail address of the corresponding author: lewis@mail.med.upenn.edu respiratory complexes. ${ }^{7}$ The prototypical heme maquette, $\mathrm{H} 10 \mathrm{H} 24$, is a de novodesigned four-helix bundle derived from the cytochrome $b c_{1}$ respiratory complex and $\alpha_{2 \mathrm{~B}}$ designed by DeGrado and co-workers. ${ }^{7-9}$ These molecules are excellent models of $b$-type cytochromes and allow us to probe the engineering tolerances of heme binding and to delineate the role of the various factors in modulating heme electrochemical function.

$\mathrm{H} 10 \mathrm{H} 24$ and related maquettes are four-helix bundles consisting of a non-covalent dimer of disulfide linked di- $\alpha$-helical subunits, $(\alpha-S S-\alpha)_{2}$. These sequences are composed mostly of three amino acid types (Glu, Lys and Leu) laid out approximately in an alternating [HPPHHPP]/ [HPPHHPH] (H: hydrophobic, P: polar) binary pattern. ${ }^{7,8}$ Replacement of Leu in the $a$ position by His10, Phe17 and His24 and in the $d$ position by Arg27, was inspired by the sequence of the cytochrome $b$ subunit of the cytochrome $b c_{1}$ complex. 
The incorporation of histidine residues provides for heme binding and the arginine residues were used to modulate the reduction potentials of the heme groups.

Structural studies of the maquettes were initially hampered by the molten globule-like folded states of both the apo and the holo forms. ${ }^{10,11}$ Many of the early de novo designed peptides that simply relied on hydrophobic sequestration to drive the folding and assembly lacked a well-defined tertiary structure $^{12-14 .}$ Although contemporary design strategies that can potentially promote structural uniqueness were devised, the early maquettes contained few of those features that would contribute to a nativelike structure. The maquette sequences had uniformly large hydrophobic residues (L, I, F) instead of alternating large and small residues in the core, which would promote complementary packing. ${ }^{13}$ Constraints, such as carefully placed disulfide bonds, ${ }^{15-18}$ were also absent in the maquette design. The maquettes do have N-terminal disulfide bonds, but they do not influence the aggregation state, packing or conformational specificity of apo maquettes (B.R.G., unpublished results). Metal binding sites have been used to define the structure of bundles. ${ }^{19,20}$ The coordination of histidine residues to heme iron in maquettes constrains bundle conformation in principle. However, the incorporation of the heme macrocycle often results in molten globule type behavior.

Gibney et al. introduced structural specificity into $\mathrm{H} 10 \mathrm{H} 24$ with two conservative hydrophobic modifications to the sequence: L6I and L13F. ${ }^{11}$ The resulting four-helix bundle scaffold has similar heme binding and redox properties as the prototype $\mathrm{H} 10 \mathrm{H} 24$ but possesses a structured hydrophobic core in the apo state. Although changing Leu to Phe at position 13 of $\mathrm{H} 10 \mathrm{H} 24$ resulted in a structured peptide, maquette design does not generally support the notion that the addition of aromatic residues promotes structural uniqueness. ${ }^{1}$ Both $\mathrm{H} 10 \mathrm{H} 24$ and a maquette variant, $\left[\mathrm{I}_{6} \mathrm{~A}_{13}\right]$ (H10H24, L6I, L13A), have four Phe and four His in the hydrophobic core, but only $\left[\mathrm{I}_{6} \mathrm{~A}_{13}\right]$ is structured.

\section{Dynamic Interfaces Immobilized in Crystal}

The structure of the covalently linked $(\alpha-$ SS- $\alpha)$ subunit was solved in solution using NMR spectroscopic methods. ${ }^{21,22}$ The structure of the di- $\alpha$-helical monomer, $(\alpha-S S-\alpha)$, was well defined using the intra- $(\alpha-S S-\alpha)$ nuclear Overhauser effects (NOEs) with clearly delineated hydrophobic and hydrophilic faces. The lack of observable inter- $(\alpha-$ SS- $\alpha)$ NOEs left the precise hydrophobic core packing scheme undetermined. ${ }^{22}$ However, a variety of analytical methods including sedimentation equilibrium analytical ultracentrifugation and ${ }^{15} \mathrm{~N}$ relaxation measurements of the molecular reorientation correlation time indicate that $\left[\mathrm{I}_{6} \mathrm{~F}_{13}\right]$ is a four-helix bundle in solution. Furthermore, the relative orientation of the two subunits, parallel $(s y n)$ or anti-parallel (anti), was ambiguous and was only recently determined to be anti by the chemical attachment of fluorescence probes and rational redesign (B.R.G., unpublished results).

We are uncertain about the range of relative motion between the di-helices at the mobile interface with the available data. However, given that all four helices of the bundle have defined mainchains and side-chains in solution, the relative movements are likely rigid-body motions. The maquette scaffold in solution is thus best described as a four-helix bundle consisting of two uniquely structured di-helices with a "slippery" interface. In the context of the crystalline state, this interface becomes fixed in a unique anti-parallel position. Herein, we present the complete four-helix bundle structure of a maquette scaffold, L31M, solved by X-ray crystallography at $2.8 \AA$ resolution. L31M is a variant of $\left[\mathrm{I}_{6} \mathrm{~F}_{13}\right]$ with a single $\mathrm{C}$-terminal modification per helix. This leucine to methionine change has minimal affect on the overall structure. The ${ }^{13} \mathrm{C}$-heteronuclear single quantum coherence (HSQC) spectrum of $\mathrm{L} 31 \mathrm{M}$ is nearly identical to the ${ }^{13} \mathrm{C}$ HSQC of $\left[\mathrm{I}_{6} \mathrm{~F}_{13}\right]$ (data not shown.) Furthermore, both $\mathrm{L} 31 \mathrm{M}$ and $\left[\mathrm{I}_{6} \mathrm{~F}_{13}\right]$ can be crystallized under the same conditions in the same space groups and unit cells.

\section{Symmetry and Topology of the Four- helix Bundle Maquette Scaffold}

The most common topology of four-helix bundles found in the RCSB database is up-down-updown, in which all helices orient opposite to their adjacent helices. Proteins such as ROP, ${ }^{23} \mathrm{PD}_{1}{ }^{24}$ Mnt repressor ${ }^{25}$ and di-zinc DF1 ${ }^{26}$ all exhibit updown-up-down topology. In principle, helix dipole and charge-charge interactions can be used to promote anti-parallel up-down-updown topology and discourage formation of parallel four-helix bundles. The maquette design did not purposely promote a specific topology. Nonetheless, placing Glu in the $b$ and $e$ positions of the maquette sequence and placing Lys in the $c$ and $g$ positions theoretically destabilizes up-up-downdown conformation and favors the "all up" topology. The arrangement of helices of L31M maquette scaffold is best described as up-updown-down (Figure 1). A31P ROP mutant ${ }^{27}$ and $\alpha_{2 \mathrm{D}}{ }^{28}$ have similar topologies. However, both structures are made of two identical helix-loop-helix units assembled in bisecting-U conformation. The loops connecting the helices in A31P ROP and $\alpha_{2 \mathrm{D}}$ cross the hydrophobic core diagonally instead connecting adjacent helices in an anti-parallel conformation. In a bisecting- $U$ arrangement, the antiparallel helices are closer together near the loop and farther apart at the termini. The anti-parallel helices in the maquette scaffold, on the other 


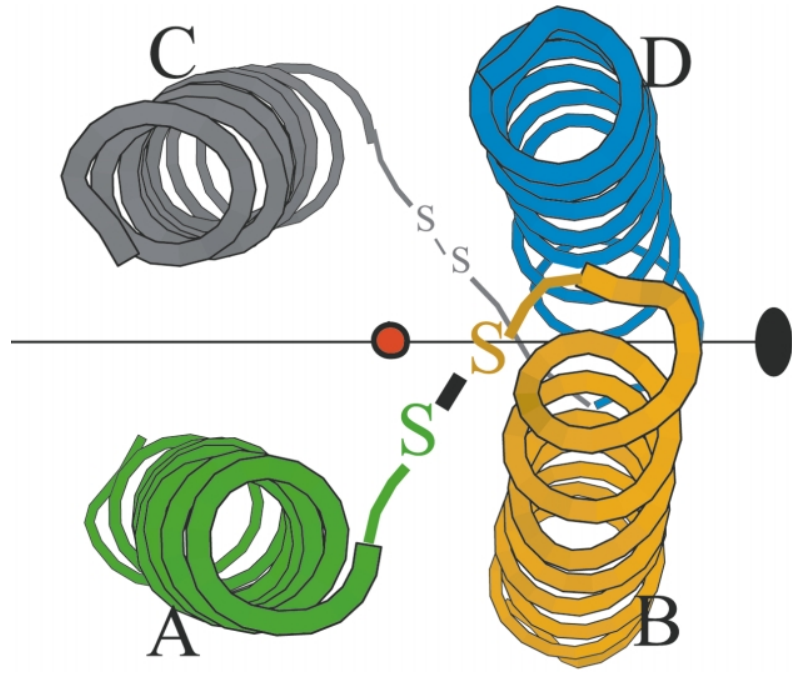

Figure 1. Axial view of the L31M four-helix bundle. Each helix is color-coded and the same color code is used in Figures 2 and 3. The $\mathrm{N}$ termini of helices $\mathrm{A}$ and $\mathrm{B}$ are toward the reader. The $C_{2}$ axis through the center of the bundle relates helices A and B to helices C and D, respectively. The red circle at the center represents the positions of the mercury atoms, which overlap in this view. The bundle displays slight right-handed supercoiling. The supercoiling is more prominent between the covalently linked helices (A and B; C and D). The Nterminal disulfide bonds are disordered in both the NMR and the crystal structures. They are schematized in the Figure to illustrate better the orientation of the helices and the connection between the helices.

hand, do not exhibit the prominent bifurcation of the bisecting- $\mathrm{U}$ structures.

L31M has $C_{2}$ symmetry; helices labeled $A$ and $C$ are symmetrically equivalent as are helices $\mathrm{B}$ and D. The three unique interfaces appear in the structure between helix A-helix C, helix B-helix D and helix A-helix B (or its symmetry equivalent helix C-helix D). For clarity, only helices A and B will be discussed, since the same applies to helices $C$ and D. No point group symmetry operator describes the relationship between the two covalently linked di-helices within the monomer subunit. However, the backbones of the A and B helices are approximately related to each other via a $94^{\circ}$ rotation plus a $1.96 \AA$ translation about an axis perpendicular to the $C_{2}$ axis $(\mathrm{rmsd}=0.4 \AA$ from residues 6 to 28). Helix $A$ is translated toward the $\mathrm{C}$ termini relative to helix $\mathrm{B}$.

The symmetry and unusual topological arrangement of the helices is not a consequence of the disulfide bonding. The methyl region of the ${ }^{13} \mathrm{C}-$ HSQC of $\left[\mathrm{I}_{6} \mathrm{~F}_{13}\right]_{4}$ (four unlinked helices) without the N-terminal cysteine or disulfide is the same as the complete $\left[\mathrm{I}_{6} \mathrm{~F}_{13}\right]_{2}$ (two $\alpha$-SS- $\alpha$ ) suggesting that the disulfide bond is not the determining factor for the up-up-down-down arrangement. Thus, four identical helices with the sequence of L31M or $\left[\mathrm{I}_{6} \mathrm{~F}_{13}\right]_{2}$ form four-helix bundles with $C_{2}$ symmetry and three distinct helix-helix interfaces.
The unusual crossing angles between the helices, which will be discussed later, do not cause the reduction in symmetry either, since the righthanded coiled coil $\mathrm{RH} 4,{ }^{29}$ which has similar helical crossing angles, possesses $C_{4}$ symmetry. The reason why four identical helices can form non-identical interfaces is that the helices A and B pack with a $1.96 \AA$ relative axial translation. The fact that there is no rotational symmetry axis between the helices $A$ and $B$ excludes the possibility that the interface between the $(\alpha-S S-\alpha)$ subunits is identical to the interface within the intra $(\alpha-S S-\alpha)$ subunit.

\section{Comparision of Crystal Di-helix Unit with the NMR Structure}

In addition to the fact that the mobile interface observed in NMR experiments is fixed in the crystal structure, there are some differences between the NMR structure of $\left[\mathrm{I}_{6} \mathrm{~F}_{13}\right](\alpha-S S-\alpha)^{22}$ and the $\mathrm{X}$-ray structure of L31M. The differences may be related to the presence of mercury in the crystal structure. The backbone rmsd between L31M and the NMR solution structure of $\left[\mathrm{I}_{6} \mathrm{~F}_{13}\right]_{2}$ is $1.23 \AA$ for residues 6-28. The axial translational offset between the helix A and helix B is similar in the crystal and in solution. A majority of the hydrophobic core side-chain conformations are nearly identical between the two structures. However, the core residue side-chain conformations $(\chi-1)$ differ at Phe13A, Leu14A, Ile6B, Leu9B, Phe13B, Phe17B, Leu20B and Leu28B. The Phe13 residues are uniformly gauche $(+)$ in the X-ray structure while trans in the NMR structure. There are two significant main-chain differences of note between the X-ray and NMR maquette scaffold structures. First, the $C$ terminus in the NMR structure's helix A turns toward helix B, whereas helix A in the $X$-ray structure remains straight relative to helix $\mathrm{B}$. The divergence is most noticeable C-terminal to His24A in the NMR structure. Second, the two structures are different between residues six and ten of helix A. The NMR structure evinces a tighter turn than a standard $\alpha$-helix, reminiscent of a $33^{-}$ helix, while the crystal structure remains $\alpha$-helical to Ile6 of helix A.

\section{The Four-helix Bundle Maquette Scaffold is not a Left-handed Coiled-coil}

There are several structures available of designed proteins with left-handed coiled-coil architectures that were based on the heptad repeat sequence pattern. ${ }^{24,26,28,30-36}$ All of these helical bundles have crossing angles near those observed in the natural left-handed coiled-coils ColE1 ROP ${ }^{23}$ and yeast GCN4. ${ }^{37}$

As designed, residues 5-31 are helical as previously shown by NMR spectroscopy. Table 1 summarizes the helical bundle parameters resulting 
Table 1. L31M mercury MAD experiment statistics

\begin{tabular}{|c|c|c|c|}
\hline Unit cell & $\begin{array}{ll}26.9 \AA & 48.8 \AA \\
90.0^{\circ} & 104.7^{\circ}\end{array}$ & $\begin{array}{l}46.7 \AA \\
90.0^{\circ}\end{array}$ & \\
\hline Space group & P2(1) & & \\
\hline Wavelength (̊̊) & 1.0019 & 1.0098 & 0.9824 \\
\hline Resolution $(\AA)$ & $20-2.7$ & $20-2.7$ & $20-2.7$ \\
\hline Redundancy & 1.76 & 1.7 & 1.7 \\
\hline$I / \sigma$ & 28.4 & 27.2 & 25.9 \\
\hline Completeness* (\%) & $97.1(88.6)$ & $96.4(85)$ & $96.1(80.6)$ \\
\hline$R_{\text {merge }} *(\%)$ & $2.8(7.2)$ & $2.9(7.2)$ & $3.1(8.5)$ \\
\hline Figure of merit* & $0.71(0.54)$ & & \\
\hline
\end{tabular}

* Numbers in parentheses are that of the last resolution ring.

from fitting to the Crick parameterization ${ }^{38}$ with the addition of two parameters that account for the helix-helix translation (values not shown). The average helix-helix separation within the $(\alpha-$ SS- $\alpha$ ) subunit is about $10.8 \AA$, which is slightly larger than those observed in a typical coiled-coil such as ROP: $9.2 \AA$ intramonomer and 10.1-10.3 $\AA$ intermonomeric distance. ${ }^{23}$ The crossing angle between the two disulfide-linked helices in the $(\alpha-$ SS- $\alpha$ ) subunit is $-5^{\circ}$, while the crossing angle between the two symmetrically related subunits is $+178^{\circ}$. The crossing angle between helix $\mathrm{A}$ and helix $\mathrm{B}$ is similar to that of right-handed coiledcoil RH4, designed with a 11-fold or undecatedrepeat sequence. ${ }^{29}$ The deviation is significant because a left-handed coiled-coil with $+20^{\circ}$ crossing angle is expected from a heptad repeat design. Dramatic changes in aggregation state and topology of helical bundles with alterations in sequence have been observed in the variants of $\mathrm{ROP}^{36}$ and GCN4. ${ }^{31}$ In both works, the crossing angles do not deviate significantly from the "wildtypes". This is expected since the 7-fold hydrophobic/hydrophilic patterns are unaltered from the respective wild-types.

The number of residues/turn in L31M is 3.6, which is consistent with $\alpha$-helix and not the 3.5 of a left-handed coiled-coil. The helical backbone, including the $C \beta$ atoms, matches closely to that of a straight polyalanine helix (rmsd $0.33 \AA$ for helix $\mathrm{A}$, and $0.4 \AA$ for helix $\mathrm{B}$ ). The difference between a 3.6 residues/turn pitch of the $\alpha$-helix and the 3.5 residues/turn pitch of a coiled-coil is apparent when attempts are made to describe L31M as a coiled-coil with a heptad repeat sequence. The positions of the $C \beta$ atom within the context of the bundle, deviate markedly from the positions expected. Based on the heptad repeat design strategy, H10, F17 and H24 are expected to be in the heptad $a$ position. However, in the structure H10 is in a heptad $a$ position, F17 occupies a location between the heptad $a$ and $d$ positions and H24 occupies a dposition. Also, expected heptad $d$ position residues F13, L20 and R27 are situated near theoretical heptad $g$ positions. Clearly, as one progresses down the helix the difference between a straight $\alpha$-helix and the expected coiled-coil become more apparent. In addition to the larger number of residues per turn, the mismatch of residue positions contributes to the clockwise crossing between helix A and helix $B$ and near-parallel arrangement between the symmetry related helices (A and C; B and D).

Residues in L31M can be modeled to have the intended position by adjusting the intra- $(\alpha-S S-\alpha)$ crossing angle to $\sim+20^{\circ}$ and by clockwise axial rotation of helices. In such a model, the side-chain of Arg27B is found buried in the core between His24A, Leu28A and Arg27A and the side-chain of Arg27A is buried between helices C and D. If the $+20^{\circ}$ crossing angle is modeled such that Arg27 is kept at the interface by rotating helices on the axes, then hydrophobic residues Ile6, Leu 9 and Phe13 would be solvent-exposed. With the $-5^{\circ}$ crossing angles, concomitant exposure of Arg27 and burial of N-terminal hydrophobic areas is possible. This may contribute to the lower energy of the $-5^{\circ}$ crossing angle compared to the $20^{\circ}$ crossing angle. It is worth mentioning that in ROP, a potentially $d$ position Arg near the $C$ terminus also exists in the sequence. Similar to Arg27 of L31M, Arg55 in ROP structure is solvent-exposed. The exposure is achieved by local distortion of the helix where Phe56 (an $e$ position by sequence) assumes the $d$ position. ${ }^{23}$ Why a similar adjustment of the backbone of L31M and $\left[\mathrm{I}_{6} \mathrm{~F}_{13}\right]_{2}$ does not occur is unclear.

\section{The Maquette Scaffold Does not Exhibit Knob-in-hole Packing}

Left handed coiled-coils employing the heptad repeat sequence pattern are expected to exhibit knob-in-hole packing. ${ }^{38}$ The $-5^{\circ}$ interhelical crossing angle found in the L31M structure cannot support systematic knob-in-hole packing which is optimal at a crossing angle of $+20^{\circ} .{ }^{38}$ Helices packed with crossing angles near $0^{\circ}$ have overlapping $C \alpha$ positions between many residues in a helical-net diagram. ${ }^{38-40}$ At the crossing angles observed in L31M, the $\mathrm{C} \alpha$ positions of residues from different coiled-coil helices overlap, preventing formation of a regular packing lattice. With certain amino acid sequences, residues from the same helix may pack with each other instead of with residues of another helix. This creates the noninterdigitated packing at the helix B-helix D interface of L31M as shown in Figure 3. Some side-chain interdigitation is observed at the $\mathrm{A}-\mathrm{B}$ (and symmetry-related C-D) interface (Figure 2) and $\mathrm{A}-\mathrm{C}$ interfaces (Figure 3 ) of L31M. The B-D interface of L31M shows little interdigitation. In contrast, knob-in-hole packing, like that observed in GCN4, shows extensive interdigitation at the packing interface. A non-interdigitated packing interface may lack structural specificity due to shortage of mutual lateral restrictions, which would have been provided by the interdigitated side-chains. It is possible that inadequate interlocking of residues may be one of the causes of the lack of structural specificity seen in early maquette scaffold designs. The lack of interdigitation between 

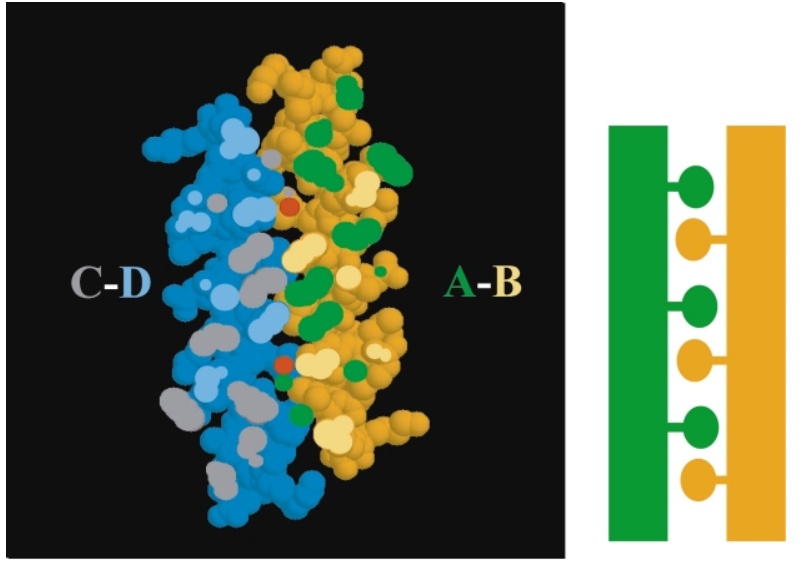

Figure 2. A slab through the center of packing interfaces between the covalently linked di-helices (RASMOL). The helices are represented by the color coding scheme in Figure 1. The red circles are mercury atoms. The diagram on the right shows a scheme of packing interface with interdigitation. An interdigitated interface in this cross-sectional representation has juxtaposition of different colors. Note, the lighter blue and orange represent the "sliced atoms" of the blue and orange helices at the cross-section.

the helices B and D of L31M may also cause the interface to be slippery, leading to the absence of NOEs at the B-D interfaces.

\section{Concluding Remarks}

Structural determination of L31M completes the first design and assessment cycle of a maquette scaf-
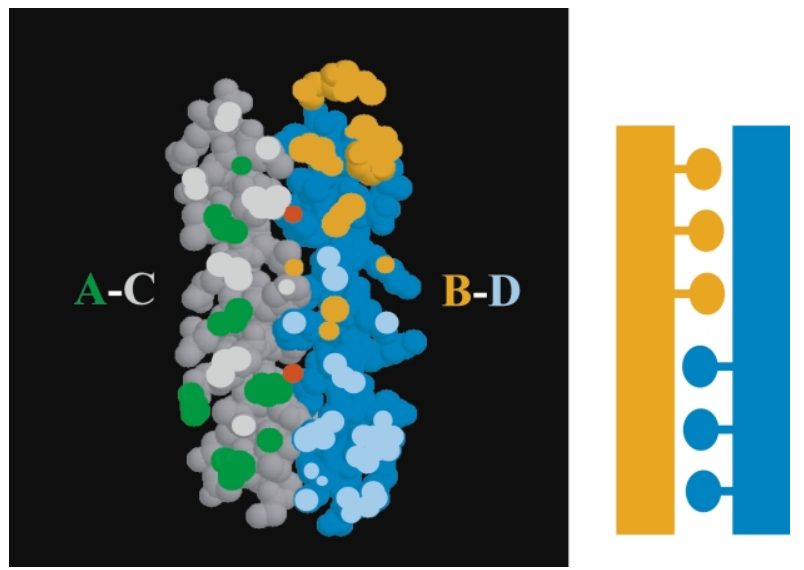

Figure 3. A slab through the center of packing interfaces between the symmetry-related helices (RASMOL). The interface between the symmetry-related B-D interface, shown in orange and blue, has fewer interdigitations than the interface between helix A and helix C. The scheme on the right shows a simplified packing arrangement between helix B and helix D illustrating the lack of interdigitation between the helices when compared to the scheme in Figure 2. Note, the lighter gray and blue represent the "sliced atoms" of the gray and blue helices at the cross-section.
Table 2. Helical parameters of the maquette structures (helixA-B)

\begin{tabular}{|c|c|c|c|c|}
\hline $\begin{array}{l}\text { Regions } \\
\text { of fit- } \\
\text { ting }\end{array}$ & $\begin{array}{l}\text { Superhelical } \\
\text { residues/ } \\
\text { turn }(2 \pi / \omega 0)\end{array}$ & $\begin{array}{l}\text { Superhelix } \\
\text { radius } \\
(R 0)(\AA)\end{array}$ & $\begin{array}{l}\mathrm{R} 1 \alpha- \\
\text { helix } \\
\text { radius } \\
(R 1)(\AA)\end{array}$ & $\begin{array}{l}\text { Superhelix } \\
\text { crossing } \\
\text { angle } \\
\text { (deg.) }\end{array}$ \\
\hline $\begin{array}{l}\text { NMR } \\
17-27\end{array}$ & 235 & 5.287 & 2.32 & 5.5 \\
\hline $\begin{array}{l}\text { X-ray } \\
17-27\end{array}$ & 285 & 5.407 & 2.25 & -4.5 \\
\hline \multirow{2}{*}{$\begin{array}{l}\text { X-ray } \\
6-17\end{array}$} & 222 & 5.25 & 2.27 & -5.6 \\
\hline & $\begin{array}{l}\text { Rise per } \\
\text { residue }(D)\end{array}$ & $\begin{array}{l}\text { Residues / } \\
\text { helical } \\
\text { turn } \\
(2 \pi / \omega 1)\end{array}$ & $\begin{array}{l}\text { RMSD of } \\
\text { fitting } \\
(\AA)\end{array}$ & \\
\hline $\begin{array}{l}\text { NMR } \\
17-27\end{array}$ & 1.47 & 3.69 & 0.46 & \\
\hline $\begin{array}{l}\text { X-ray } \\
17-27\end{array}$ & 1.507 & 3.58 & 0.25 & \\
\hline $\begin{array}{l}\text { X-ray } \\
6-17\end{array}$ & 1.513 & 3.59 & 0.27 & \\
\hline
\end{tabular}

Crossing angle $=\operatorname{ArcSin}\left(R_{0} \omega_{0} / d\right)$

fold. We learned that helices with crossing angles near $0^{\circ}$ must be considered in the design especially when the sequence contains many large hydrophobic residues. We also realize that symmetry in the design clearly depends on whether or not the most stable conformation preserves the structural identity among helices. Identical helices need not generate identical interfaces. Furthermore, not all interfaces need to be extensively interdigitated for the structure to be unique in crystalline state. Careful adjustment of the residues at the interface can alter considerably the association at the interfaces. The design history of $\left[\mathrm{I}_{6} \mathrm{~F}_{13}\right]$ and L31M suggests that one may iteratively fine-tune the packing at each interface. The L6I and L13F amino acid changes that are responsible for the conformational specificity of $\left[\mathrm{I}_{6} \mathrm{~F}_{13}\right]$ are found at the $\mathrm{A}-\mathrm{B}(\mathrm{C}-\mathrm{D})$ and $\mathrm{A}-\mathrm{C}$ interfaces but not the $B-D$ interface; the $A-B, C-D$ and $A-C$ interfaces have more observed interdigitation (Figs. 2 and 3) than the B-D interface (Figure 3). With modifications at positions such as 14, 21 and 28 , which are at the B-D interface, the amount of interdigitation between the helices may increase, leading to the achievement of structural specificity between the di-helices.

\section{Material and Methods}

\section{Crystallization and phasing}

We replaced L31 in $\left[\mathrm{I}_{6} \mathrm{~F}_{13}\right]$ with methionine early in the study to gain the option of selenomethionine multiple anomalous dispersion (MAD) studies. The sequence of L31M is (Ac-CGGG EI $\mathrm{EI}_{6} \mathrm{WK}-\mathrm{H}_{10} \mathrm{EEF}_{13}$ LKK-FEELLKLFEERLKK-M $\left.{ }_{31}-\mathrm{CONH}_{2}\right)$. The $\mathrm{N}$ termini are acetylated and the C-termini are amidated. Synthesis and purification of the peptides are detailed elsewhere. ${ }^{41}$ Both L31M and $\left[\mathrm{I}_{6} \mathrm{~F}_{13}\right]$ can be crystallized under the same conditions in the same unit cell of $a=26.9 \AA, b=48.8 \AA, c=46.7 \AA$, 
$\alpha=90.0^{\circ}, \beta=104.7^{\circ}, \gamma=90.0^{\circ}$ in $P 2_{1}$ space group. The optimal crystallization samples are HPLC pure L31M ( $\alpha$ SS- $\alpha)_{2}$ peptides dialyzed against deionized water and concentrated to $0.6 \mathrm{mM}$ four-helix bundle. The crystals were formed from hanging-drop over a reservoir containing $30 \%(\mathrm{w} / \mathrm{v})$ PEG2000 monomethylether and 0.1 M Hepes at $\mathrm{pH}$ 7.4. The crystallization drop itself contained a mixture of 2:5 peptide:reservoir. Crystals emerged within one to three days at $28{ }^{\circ} \mathrm{C}$ and were grown to $200 \mu \mathrm{m} \times 100$ $\mu \mathrm{m} \times 50 \mu \mathrm{m}$ over an additional 24 hours. The crystals were conditioned with cryoprotectant (30\% PEG550 buffered with $30 \mathrm{mM}$ Hepes at $\mathrm{pH}$ 7.4) before they were frozen in a liquid nitrogen stream for storage or for data collection. For heavy atom derivitization, the crystals adapted to the cryoprotectant were soaked in a solution consisting of $30 \mathrm{mM}$ ethylmercuryphosphate in $30 \%$ PEG550 for 12 hours before freezing. A MAD data set was collected at Beamline X12C at the National Synchrontron Light Source on a Brandeis CCD detector at the mercury LIII edge (1.0019 $\AA$ ), the inflection point $(0.9824 \AA)$, and a remote wavelength $(1.0098 \AA)$. Data integration and reduction were performed with DENZO. ${ }^{42}$ The initial phase was calculated with SOLVE ${ }^{43}$ and solvent flattened with RESOLVE. ${ }^{44,45}$ Structural analysis and verification were performed with PROCHECK. ${ }^{46}$

\section{Refinement}

The backbone of the four-helix bundle is visible from residues 3-31 with the $\mathrm{C} \alpha$ positions of these residues clearly defined. However, many solvent-exposed amino acid side-chains, particularly Glu and Lys, are not visible beyond their C $\beta$ s. Mercury density is located within the hydrophobic core near the His24s. Model building used $\mathrm{O}^{47}$ followed by refinement with CNS. ${ }^{48}$ Refinements that minimize the standard crystallographic residual did not converge because the data (unique reflections) to variable (number of non-hydrogen atoms multiplied by four) ratio is only about 0.7 at $2.8 \mathrm{~A}$. However, refinements were successful when phase restraints and non-crystallographic symmetry (NCS) restraints were applied simultaneously. The phase restraint is applied by using the "mixed" target for refinement. The final $R=23.4 \%$ and $R_{\mathrm{f}}=27.8 \%$. The native data sets of $\mathrm{L} 31 \mathrm{M},\left[\mathrm{I}_{6} \mathrm{~F}_{13}\right]_{2}$ and other maquette crystals (S.S.H., unpublished results) have not be refined to satisfaction because there is no additional phase information at this time due to lack of high resolution data and phase restraints. Table 2 summarizes the MAD experiment and the refinement statistics for L31M.

Within the crystal structure there is a questionable $x-1$ torsion angle: Phe17 of the B helix. The density to which the structure was fitted is incomplete, and the phenyl ring has high temperature factors $\left(B>40 \AA^{2}\right.$.) The $\chi$ - 1 angle of Phe17B in the structure is $230^{\circ}$, which is mid-way between two favorable angles $\left(183^{\circ}\right.$ and $\left.-66^{\circ}\right)$, suggesting that Phe17B exists in two equally favorable conformations. The fitted Phe17B conformation is likely the average of positions of Phe17B. Note that Phe17 of the helix B in the NMR structure is in the allowed region of trans conformation with $\chi-1$ approximately $-160^{\circ}$ (or $200^{\circ}$ ).

\section{Atomic coordinates}

The coordinates have been deposited into the RCSB database with accession code PDB ID 1M3W.

\section{Acknowledgements}

This work was supported by grants from the National Institutes of Health (GM 48130 and GM 41048 to P.L.D.; Cell and Molecular Biology Training Grant to S.S.H.). The MAD data set was collected during the RapiData 2000 course at Brookhaven National Laboratory. Mass spectroscopic analyses were performed by the Protein Chemistry Laboratory of the University of Pennsylvania.

\section{References}

1. Baltzer, L., Nilsson, H. \& Nilsson, J. (2001). De novo design of proteins-what are the rules? Chem. Rev. 101, 3153-3163.

2. Beasley, J. R. \& Hecht, M. H. (1997). Protein design: the choice of de novo sequences. J. Biol. Chem. 272, 2031-2034.

3. Bryson, J. W., Betz, S. F., Lu, H. S., Suich, D. J., Zhou, H. X. X., Oneil, K. T. \& Degrado, W. F. (1995). Protein design-a hierarchical approach. Science, 270, 935-941.

4. Hellinga, H. W. (1998). The construction of metal centers in proteins by rational design. Fold. Des. 3, R1-R8.

5. Kennedy, M. L. \& Gibney, B. R. (2001). Metalloprotein and redox protein design. Curr. Opin. Struct. Biol. 11, 485-490.

6. Moffet, D. A. \& Hecht, M. H. (2001). De novo proteins from combinatorial libraries. Chem. Rev. 101, 3191-3203.

7. Robertson, D. E., Farid, R. S., Moser, C. C., Urbauer, J. L., Mulholland, S. E., Pidikiti, R. et al. (1994). Design and synthesis of multi-heme proteins. Nature, 368, 425-431.

8. Degrado, W. F., Wasserman, Z. R. \& Lear, J. D. (1989). Protein design, a minimalist approach. Science, 243, 622-628.

9. Ho, S. P. \& Degrado, W. F. (1987). Design of a 4-helix bundle protein-synthesis of peptides which selfassociate into a helical protein. J. Am. Chem. Soc. 109, 6751-6758.

10. Gibney, B. R., Rabanal, F., Reddy, K. S. \& Dutton, P. L. (1998). Effect of four helix bundle topology on heme binding and redox properties. Biochemistry, 37, 4635-4643.

11. Gibney, B. R., Rabanal, F., Skalicky, J. J., Wand, A. J. \& Dutton, P. L. (1997). Design of a unique protein scaffold for maquettes. J. Am. Chem. Soc. 119, 2323-2324.

12. Betz, S. F., Raleigh, D. P. \& Degrado, W. F. (1993). De Novo protein design-from molten globules to native-like states. Curr. Opin. Struct. Biol. 3, 601-610.

13. Munson, M., Balasubramanian, S., Fleming, K. G., Nagi, A. D., O'Brien, R., Sturtevant, J. M. \& Regan, L. (1996). What makes a protein a protein? Hydrophobic core designs that specify stability and structural properties. Protein Sci. 5, 1584-1593.

14. Munson, M., O'Brien, R., Sturtevant, J. M. \& Regan, L. (1994). Redesigning the hydrophobic core of a 4helix-bundle protein. Protein Sci. 3, 2015-2022.

15. Hecht, M. H., Richardson, J. S., Richardson, D. C. \& Ogden, R. C. (1990). De novo design, expression and characterization of FELIX - a 4-helix bundle protein of native-like sequence. Science, 249, 884-891. 
16. Kuroda, Y., Nakai, T. \& Ohkubo, T. (1994). Solution structure of a de novo helical protein by 2D-NMR spectroscopy. J. Mol. Biol. 236, 862-868.

17. Myszka, D. G. \& Chaiken, I. M. (1994). Design and characterization of an intramolecular antiparallel coiled-coil peptide. Biochemistry, 33, 2363-2372.

18. Zhou, N. E., Kay, C. M. \& Hodges, R. S. (1993). Disulfide bond contribution to protein stability-positional effects of substitution in the hydrophobic core of the 2-stranded alpha-helical coiled-coil. Biochemistry, 32, 3178-3187.

19. Handel, T. \& Degrado, W. F. (1990). De novo design of a Zn-2 + -binding protein. J. Am. Chem. Soc. 112, 6710-6711.

20. Regan, L. \& Clarke, N. D. (1990). A tetrahedral zinc(Ii)-binding site introduced into a designed protein. Biochemistry, 29, 10878-10883.

21. Skalicky, J. J., Bieber, R. J., Gibney, B. R., Rabanal, F., Dutton, P. L. \& Wand, A. J. (1998). Sequence-specific resonance assignments for a designed four-alphahelix bundle protein. J. Biomol. NMR, 11, 227-228.

22. Skalicky, J. J., Gibney, B. R., Rabanal, F., Urbauer, R. J. B., Dutton, P. L. \& Wand, A. J. (1999). Solution structure of a designed four-alpha-helix bundle maquette scaffold. J. Am. Chem. Soc. 121, 4941-4951.

23. Banner, D. W., Kokkinidis, M. \& Tsernoglou, D. (1987). Structure of the Cole1 Rop protein at $1.7 \AA$ resolution. J. Mol. Biol. 196, 657-675.

24. Schafmeister, C. E., Miercke, L. J. W. \& Stroud, R. M. (1993). Structure at 2.5 Angstrom of a designed peptide that maintains solubility of membrane-proteins. Science, 262, 734-738

25. Nooren, I. M. A., Kaptein, R., Sauer, R. T. \& Boelens, R. (1999). The tetramerization domain of the Mnt repressor consists of two right-handed coiled coils. Nature Struct. Biol. 6, 755-759.

26. Lombardi, A., Summa, C. M., Geremia, S., Randaccio, L., Pavone, V. \& DeGrado, W. F. (2000). Retrostructural analysis of metalloproteins: application to the design of a minimal model for diiron proteins. Proc. Natl Acad. Sci. USA, 97, 6298-6305.

27. Glykos, N. M., Cesareni, G. \& Kokkinidis, M. (1999). Protein plasticity to the extreme: changing the topology of a 4-alpha-helical bundle with a single amino acid substitution. Struct. Fold. Des. 7, 597-603.

28. Hill, R. B. \& DeGrado, W. F. (1998). Solutions structure of alpha D-2, a native-like de novo designed protein. J. Am. Chem. Soc. 120, 1138-1145.

29. Harbury, P. B., Plecs, J. J., Tidor, B., Alber, T. \& Kim, P. S. (1998). High-resolution protein design with backbone freedom. Science, 282, 1462-1467.

30. Harbury, P. B., Kim, P. S. \& Alber, T. (1994). Crystalstructure of an isoleucine-zipper trimer. Nature, 371, 80-83.

31. Harbury, P. B., Zhang, T., Kim, P. S. \& Alber, T. (1993). A switch between 2-stranded, 3-stranded and 4stranded coiled coils in gen4 leucine-zipper mutants. Science, 262, 1401-1407.

32. Lovejoy, B., Choe, S., Cascio, D., McRorie, D. K., Degrado, W. F. \& Eisenberg, D. (1993). Crystal-struc- ture of a synthetic triple-stranded alpha-helical bundle. Science, 259, 1288-1293.

33. Ogihara, N. L., Weiss, M. S., Degrado, W. F. \& Eisenberg, D. (1997). The crystal structure of the designed trimeric coiled coil coil-V(a)L(d): implications for engineering crystals and supramolecular assemblies. Protein Sci. 6, 80-88.

34. Schafmeister, C. E., LaPorte, S. L., Miercke, L. J. W. \& Stroud, R. M. (1997). A designed four helix bundle protein with native-like structure. Nature Struct. Biol. 4, 1039-1046.

35. Walsh, S. T. R., Cheng, H., Bryson, J. W., Roder, H. \& DeGrado, W. F. (1999). Solution structure and dynamics of a de novo designed three-helix bundle protein. Proc. Natl Acad. Sci. USA, 96, 5486-5491.

36. Willis, M. A., Bishop, B., Regan, L. \& Brunger, A. T. (2000). Dramatic structural and thermodynamic consequences of repacking a protein's hydrophobic core. Structure, 8, 1319-1328.

37. O'shea, E. K., Klemm, J. D., Kim, P. S. \& Alber, T. (1991). X-Ray Structure of the Gcn4 Leucine Zipper, a 2-Stranded, Parallel Coiled Coil. Science, 254, 539-544.

38. Crick, F. H. C. (1953). The packing of $\alpha$-helices: simple coiled-coils. Acta Crystallog. 6, 689-697.

39. Chothia, C., Levitt, M. \& Richardson, D. (1981). Helix to helix packing in proteins. J. Mol. Biol. 145, 215-250.

40. Walther, D., Eisenhaber, F. \& Argos, P. (1996). Principles of helix-helix packing in proteins: the helical lattice superposition model. J. Mol. Biol. 255, $536-553$.

41. Gibney, B. R., Rabanal, F., Skalicky, J. J., Wand, A. J. \& Dutton, P. L. (1999). Iterative protein redesign. J. Am. Chem. Soc. 121, 4952-4960.

42. Otwinowski, Z. \& Minor, W. (1997). Processing of X-ray diffraction data collected in oscillation mode. Methods Enzymol., 276, 307-326.

43. Terwilliger, T. C. \& Berendzen, J. (1999). Automated MAD and MIR structure solution. Acta Crystallog. sect. D-Biol. Crystallog. 55, 849-861.

44. Terwilliger, T. C. (1999). Reciprocal-space solvent flattening. Acta Crystallog. Sect. D-Biol. Crystallog. 55, 1863-1871.

45. Terwilliger, T. C. (2000). Maximum-likelihood density modification. Acta Crystallog. Sect. D-Biol. Crystallog. 56, 965-972.

46. Laskowski, R. A., MacArthur, M. W., Moss, D. S. \& Thornton, J. M. (1993). PROCHECK: a program to check the stereochemical quality of protein structures. J. Appl. Crystallog. 26, 283-291.

47. Jones, T. A., Zou, J. Y., Cowan, S. W. \& Kjeldgaard, M. (1991). Improved methods for building protein models in electron-density maps and the location of errors in these models. Acta Crystallog. sect., A, 47, 110-119.

48. Brunger, A. T., Adams, P. D., Clore, G. M., DeLano, W. L., Gros, P., Grosse-Kunstleve, R. W. et al. (1998). Crystallography and NMR system: a new software suite for macromolecular structure determination. Acta Crystallog. Sect. D-Biol. Crystallog. 54, 905-921. 